\title{
Computed tomographic quantification of periaortic adipose tissue volume as a correlate of cardiovascular disease
}

\author{
Nathan Robbins ${ }^{1,2}$, Edmond A. Hooker ${ }^{1}$, Kim W. Hart ${ }^{1}$, \\ Sangita Kapur ${ }^{3}$, Andra Blomkalns ${ }^{4}$ \\ ${ }^{1}$ Department of Emergency Medicine, College of Medicine, University of Cincinnati, United States \\ ${ }^{2}$ Heritage College of Osteopathic Medicine, Ohio University, United States \\ ${ }^{3}$ Department of Radiology, College of Medicine, University of Cincinnati, United States \\ ${ }^{4}$ Department of Emergency Medicine, UT Southwestern Medical Center, United States
}

The accumulation of adipose tissue has long been thought of as a risk factor for cardiovascular disease (CVD) [1]. However, the dynamic effects in metabolic homeostasis and pathology are still being discovered [2]. The complexity of factors associated with obesity including adipokines associated with various adipose tissue depots is frequently overlooked [3]. Intuitively, one of these depots, perivascular adipose tissue (PVAT), is likely contributing to the development and/or progression of CVD given its proximity to arterial vasculature. A subset of PVAT that surrounds the coronary arteries, epicardial fat, has been well studied in the development of coronary artery disease [4]. Another subset of PVAT, periaortic adipose tissue has also been implicated in CVD, however, previous studies have limited their investigation to a specific population, unique depots, or a single CVD [5-7]. Herein, the current study describes the quantification of periaortic adipose tissue volume in both the thoracic and abdominal regions in a unique population and correlates this value to specific measures of CVD.

Once the study protocol and procedures were approved by the University of Cincinnati Institutional Review Board (IRB\#2013-8286), An electronic medical record was queried for patients who obtained non-contrasted computed tomography (CT) scans of their chest and/or abdomen over a 2-year period. These deidentified records were reviewed to verify subjects met inclusion/exclusion criteria which were broad, allowing for a diverse patient population. Inclusion criteria included 18 years of age or older and non-contrasted CT scan of chest and/or abdomen. Exclusion criteria where limited to variables that would compromise the measurement of the periaortic tissue volume including previous aortic surgery, gross anatomical anomalies (including trauma) or the use of contrast as the increased attenuation within the lumen would alter the radiodensity of the surrounding tissue.

After appropriate subjects were identified, abdominal and thoracic periaortic adipose tissue volume was quantified in a similar manner as previously conducted [5]. The segment of thoracic aorta that was measured started at the level of the pulmonary artery bifurcation and extended $70 \mathrm{~mm}$ inferiorly. The abdominal aortic segment was measured starting at the level of the aortic bifurcation and extended superiorly $40 \mathrm{~mm}$. Adipose tissue was selectively gated using a window width of -195 to -45 Hounsfield units (HU) with a center of $-120 \mathrm{HU}$ [5]. The region of interest was encircled with a diameter being $10 \mathrm{~mm}$ larger than the anteroposterior aortic diameter and then adipose tissue was selectively gated [6]. The degree of aortic calcification was determined by the volume of hyperattenuation with a minimum of three connected pixels with attenuation over $130 \mathrm{HU}$ [5].

Address for correspondence: Nathan Robbins, MS, Department of Emergency Medicine, University of Cincinnati College of Medicine, 231 Albert Sabin Way, Cincinnati, Oh 45267, United States, tel: 513-502-1934, fax: 513-558-3487, e-mail: robbinnn@email.uc.edu

Received: 11.06.2019 Accepted: 3.11.2019 


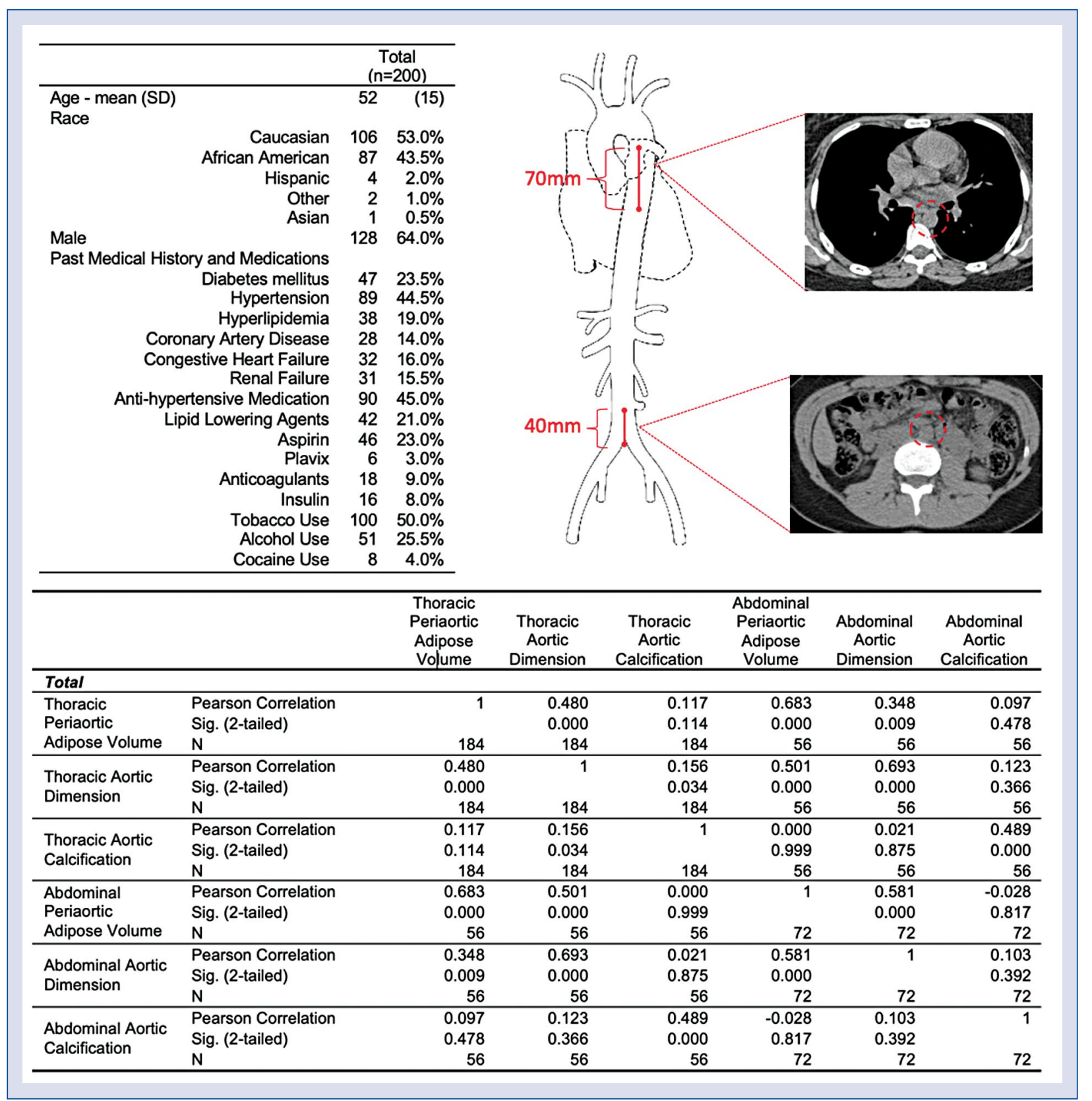

Figure 1. Summary of patient demographics, methods and results. Top left: Demographic information of the study population; Top right: Representative diagram of computed tomographic methods and periaortic adipose tissue volume quantification; Bottom: Summary of the Pearson correlations and significance of periaortic adipose tissue volume in the thoracic and abdominal regions to aortic dimension and aortic calcification in the same regions.

The aortic dimension was calculated as the mean of the anteroposterior and transverse diameter of the aorta from the outer edge to the outer edge at the level of the right pulmonary artery for the thoracic aorta and $5 \mathrm{~cm}$ above the aortoiliac bifurcation for the mid-abdominal aorta. A representation of the regions can be seen in Figure 1 (top right). All scans were performed on a Siemens SOMATOM scanner. Study data were collected and managed using Research Electronic Data Capture (REDCap) electronic data capture tools [8]. All statistical analyses were conducted using SPSS 24.0 (IBM Corporation, Armonk, NY). Data is as the median with minimum and maximum reported. Pearson correlation was calculated between periaortic adipose tissue volume in the thoracic and abdominal regions with aortic dimension and calcification in the same regions. 
Two hundred cases were reviewed, 184 noncontrast chest $\mathrm{CT}$ s and 72 non-contrast abdominal CTs. A detailed list of this demographic information is shown in Figure 1 (top left). Thoracic perivascular adipose tissue volume (PVAT) correlated the best with abdominal PVAT with an $r$ statistic of $0.683(\mathrm{p}<0.000)$. Thoracic PVAT also correlated well with thoracic aortic dimension $(r=0.480$; $\mathrm{p}<0.000$ ). Similarly, abdominal PVAT also correlated with thoracic and abdominal aortic dimension $(\mathrm{r}=0.501 ; \mathrm{p}<0.000$ and $\mathrm{r}=0.581 ; \mathrm{p}<0.000$, respectively). A summary of the data can be seen in the table at the bottom of Figure 1.

In the current study, two distinct regions of the aorta, thoracic and abdominal, were analyzed to quantify PVAT volume via selective gating for adipose tissue. These values were correlated with surrogates of CVD including aortic dimension and aortic calcification. It was found that PVAT volume correlated well with the aortic dimension in both thoracic and abdominal regions. This data is congruent with previously publish studies investigating similar endpoints including CVD risk factors and abdominal aortic aneurysms [5-7].

In conclusion, periaortic adipose tissue volume was higher in individuals with CVD in both the thoracic and abdominal regions, albeit more so around the thoracic aorta. While this association is significant, the direct clinical relevance of these specific depot volumes is still to be determined; therefore, further work needs to be conducted to investigate the association of periaortic adipose volume with cardiovascular outcomes.

Conflict of interest: None declared

\section{References}

1. Larsson B, Svärdsudd K, Welin L, et al. Abdominal adipose tissue distribution, obesity, and risk of cardiovascular disease and death: 13 year follow up of participants in the study of men born in 1913. Br Med J (Clin Res Ed). 1984; 288(6428): 1401-1404, doi: 10.1136/bmj.288.6428.1401, indexed in Pubmed: 6426576.

2. Kong Yi, Zhang S, Wu R, et al. New insights into different adipokines in linking the pathophysiology of obesity and psoriasis. Lipids Health Dis. 2019; 18(1): 171, doi: 10.1186/s12944-0191115-3, indexed in Pubmed: 31521168.

3. Kershaw EE, Flier JS. Adipose tissue as an endocrine organ. J Clin Endocrinol Metab. 2004; 89(6): 2548-2556, doi: 10.1210/ jc.2004-0395, indexed in Pubmed: 15181022.

4. Yorgun H, Canpolat U, Hazırolan T, et al. Epicardial adipose tissue thickness predicts descending thoracic aorta atherosclerosis shown by multidetector computed tomography. Int J Cardiovasc Imaging. 2012; 28(4): 911-919, doi: 10.1007/s10554-011-9899-x, indexed in Pubmed: 21637979.

5. Lehman SJ, Massaro JM, Schlett CL, et al. Peri-aortic fat, cardiovascular disease risk factors, and aortic calcification: the Framingham Heart Study. Atherosclerosis. 2010; 210(2): 656661, doi: 10.1016/j.atherosclerosis.2010.01.007, indexed in Pubmed: 20152980.

6. Schlett CL, Massaro JM, Lehman SJ, et al. Novel measurements of periaortic adipose tissue in comparison to anthropometric measures of obesity, and abdominal adipose tissue. Int J Obes (Lond). 2009; 33(2): 226-232, doi: 10.1038/ijo.2008.267, indexed in Pubmed: 19139753.

7. Dias-Neto M, Meekel JP, van Schaik TG, et al. High density of periaortic adipose tissue in abdominal aortic aneurysm. Eur J Vasc Endovasc Surg. 2018; 56(5): 663-671, doi: 10.1016/j. ejvs.2018.07.008, indexed in Pubmed: 30115505.

8. Harris PA, Taylor R, Thielke R, et al. Research electronic data capture (REDCap) - a metadata-driven methodology and workflow process for providing translational research informatics support. J Biomed Inform. 2009; 42(2): 377-381, doi: 10.1016/j. jbi.2008.08.010, indexed in Pubmed: 18929686. 